\title{
Ginsenoside Rg2 protects cardiomyocytes against trastuzumab-induced toxicity by inducing autophagy
}

\author{
GUANG LIU ${ }^{1}$, XIAOYONG $\mathrm{QI}^{2},{\mathrm{XINGTAO} \mathrm{LI}^{3} \text { and FANGYI SUN }}^{3}$ \\ ${ }^{1}$ Department of Cardiovascular Medicine, Hebei Medical University; ${ }^{2}$ Department of Cardiovascular Medicine, \\ Hebei General Hospital; ${ }^{3}$ Department of Cardiovascular Medicine, The Fourth Affiliated Hospital of \\ Hebei Medical University, Shijiazhuang, Hebei 050000, P.R. China
}

Received August 9, 2019; Accepted February 25, 2020

DOI: $10.3892 /$ etm.2021.9904

\begin{abstract}
Trastuzumab (TZM) significantly improves the outcomes of patients with breast cancer; however, it is associated with severe cardiotoxicity. Ginsenoside $\mathrm{Rg} 2$ was reported to exert protective effects against myocardial injury and apoptosis in human cardiomyocytes (HCMs). However, whether ginsenoside Rg2 protects HCMs against TZM-induced toxicity remains unclear. The present study investigated the proliferation of HCMs using a Cell Counting Kit-8 assay and Ki67 immunofluorescence staining. Apoptotic cells were detected by Annexin V/propidium iodide staining and flow cytometry. Furthermore, monodansylcadaverine staining was performed to detect cell autophagy. In addition, western blotting was used to detect the expression levels of phosphorylated (p)-Akt, p-mTOR, beclin 1 , microtubule associated protein 1 light chain $3 \alpha$ (LC3) and autophagy protein 5 (ATG5) in HCMs. Pretreatment with ginsenoside $\mathrm{Rg} 2$ significantly protected HCMs against TZM-induced cytotoxicity by inhibiting apoptosis. Furthermore, pretreatment with ginsenoside Rg2 induced autophagy in HCMs by upregulating the expression levels of p-Akt, p-mTOR, beclin 1, LC3 and ATG5. The results obtained in the present study suggested that ginsenoside Rg2 could protect HCMs against TZM-induced cardiotoxicity by activating autophagy. Therefore, ginsenoside Rg2 may serve as a potential therapeutic agent to prevent TZM-related cardiotoxicity in patients with breast cancer.
\end{abstract}

\section{Introduction}

Ginseng (Panax ginseng) is a traditional Chinese herbal medicine that has been widely used for thousands of years in East Asia, including China, Korea and Bhutan (1). The main components responsible for its therapeutic effects are ginsenosides, which

Correspondence to: Dr Xiaoyong Qi, Department of Cardiovascular Medicine, Hebei General Hospital, 348 West Heping Road, Shijiazhuang, Hebei 050000, P.R. China

E-mail: xiaoyongqi28@126.com

Key words: trastuzumab, ginsenoside $\operatorname{Rg} 2$, apoptosis, autophagy are divided into protopanaxadiol and protopanaxatriol ginsenoside groups (2). Ginsenoside $\mathrm{Rg} 2$ is one of the compounds in the protopanaxatriol group (3). Ginsenoside $\mathrm{Rg} 2$ improved neurological performance and enhanced memory by inhibiting neuronal apoptosis in a rat model of vascular dementia (3). Ginsenoside $\mathrm{Rg} 2$ improved neuronal and metabolic activities by inducing autophagy in a protein kinase AMP-activated catalytic subunit $\alpha 2$ (AMPK)/unc-51 like autophagy activating kinase 1-dependent and mTOR-independent manner (4). Furthermore, ginsenoside Rg2 increased autophagy in MCF-7 cells in vitro (5). Additionally, it was reported that ginsenoside $\mathrm{Rg} 2$ exhibited protective effects against hydrogen peroxide-induced injury and apoptosis in human cardiomyocytes (HCMs) (6).

Trastuzumab (TZM) is a humanized monoclonal antibody that targets the extracellular domain of human epidermal growth factor receptor 2 (HER2) and exerts significant therapeutic effects in early-stage HER2-positive breast cancer (7). TZM significantly improves the overall survival of the majority of patients with HER2-positive breast cancer (8). However, despite its beneficial effects, TZM is associated with several cardiac side effects, including congestive heart failure, hypertension, thromboembolic disease, ischemic heart disease, QT prolongation and bradycardia $(9,10)$.

The present study aimed to investigate whether ginsenoside $\mathrm{Rg} 2$ could protect HCMs against TZM-induced toxicity in vitro. The results may improve the care of patients with breast cancer treated with TZM.

\section{Materials and methods}

Cell culture. Human primary HCMs (Applied Biological Materials, Inc.) were cultured in DMEM (Sigma-Aldrich; Merck KGaA) containing 10\% FBS (Thermo Fisher Scientific, Inc.) and $1 \%$ streptomycin-penicillin, and maintained at $37^{\circ} \mathrm{C}$ and $5 \% \mathrm{CO}_{2}$. Upon reaching $80 \%$ confluency, HCMs were pretreated with $200 \mu \mathrm{M}$ ginsenoside Rg2 [purity $>98 \%$ (by high-performance liquid chromatography analysis); MedChemExpress] for $12 \mathrm{~h}$ at $37^{\circ} \mathrm{C}$, followed by exposure to $100 \mu \mathrm{g} / \mathrm{ml} \mathrm{TZM} \mathrm{(Roche} \mathrm{Diagnostics)} \mathrm{for} 24 \mathrm{~h}$ at $37^{\circ} \mathrm{C}$.

Cell proliferation assay. HCMs $\left(5 \times 10^{3}\right.$ cells/well) were seeded in a 96-well plate in triplicate. The cells were subsequently treated with $\operatorname{TZM}(0,10,50,100$ or $200 \mu \mathrm{g} / \mathrm{ml})$, 
ginsenoside $\operatorname{Rg} 2(0,50,100,200$, or $300 \mu \mathrm{M})$, a combination of $200 \mu \mathrm{M}$ ginsenoside $\mathrm{Rg} 2$ and $100 \mu \mathrm{g} / \mathrm{ml} \mathrm{TZM}$ or a combination of ginsenoside $200 \mu \mathrm{M} \mathrm{Rg} 2,100 \mu \mathrm{g} / \mathrm{ml} \mathrm{TZM}$ and $5 \mathrm{mM}$ 3-methyladenine (3-MA; Sigma-Aldrich; Merck KGaA) for $24 \mathrm{~h}$ at $37^{\circ} \mathrm{C}$. Following treatment, the cells were further incubated with $10 \mu$ l Cell Counting Kit-8 (CCK-8) solution (Dojindo Molecular Technologies, Inc.) at $37^{\circ} \mathrm{C}$ for $3 \mathrm{~h}$ and the absorbance was measured at a wavelength of $490 \mathrm{~nm}$ using a microplate reader. The cells in the control group were not given any treatment. $\mathrm{IC}_{50}$ values were determined using GraphPad Prism software (version 7.0; GraphPad Software, Inc.).

Apoptosis assay. Cell apoptosis was detected using a propidium iodide (PI) and Annexin V staining kit (BD Biosciences) according to the manufacturer's protocol. Briefly, $2 \times 10^{5} \mathrm{HCMs}$ were seeded in a 6 -well plate and then treated with 50 or $100 \mu \mathrm{g} / \mathrm{ml}$ TZM for $24 \mathrm{~h}$ and were subsequently harvested and washed twice with cold PBS. Subsequently, the cells were resuspended in binding buffer and stained with $2 \mu \mathrm{l}$ Annexin V and $2 \mu \mathrm{l}$ PI for $15 \mathrm{~min}$ at $25^{\circ} \mathrm{C}$ in the dark. The cell apoptosis rate was determined using a FACSCanto II flow cytometer (BD Biosciences) and BD CellQuest ${ }^{\mathrm{TM}}$ Pro software (version 5.1; BD Biosciences).

Western blotting. HCMs were rinsed and lysed using RIPA lysis buffer (EMD Millipore) containing a protease and phosphatase inhibitor. Subsequently, the cell lysates were vortexed on ice five times within $20 \mathrm{~min}$ and centrifuged for $10 \mathrm{~min}$ at $10,000 \mathrm{xg}$ at $4^{\circ} \mathrm{C}$. The protein concentration was determined using a bicinchoninic acid protein quantification kit (Promega Corporation). Protein aliquots $(30 \mu \mathrm{g})$ were subjected to SDS-PAGE on a $10 \%$ gel and subsequently transferred onto PVDF membranes (EMD Millipore). The membranes were blocked using $5 \%$ non-fat milk for $1 \mathrm{~h}$ at room temperature. The membranes were then incubated with the following primary antibodies: Anti-phosphorylated (p)-AKT (cat. no. ab38449), anti-p-mTOR (cat. no. ab84400), anti-autophagy protein 5 (ATG5, cat. no. ab109490), anti-beclin 1 (cat. no. ab210498), anti-microtubule associated protein 1 light chain $3 \alpha$ (LC3, cat. no. ab62721) overnight at $4^{\circ} \mathrm{C}$. All primary antibodies were used at a 1:200 dilution and were purchased from Abcam. Following washing with PBS, the membranes were incubated with horseradish peroxidase-conjugated secondary antibodies (Abcam) for $1 \mathrm{~h}$ at room temperature. An ECL reagent kit (Santa Cruz Biotechnology, Inc.) was used to visualize the immunoreactive bands according to the manufacturer's protocol. Protein band intensities were quantified using ImageJ software (v1.8.0.112; National Institutes of Health). $\beta$-actin (1:200 dilution; cat. no. ab8227; Abcam) acted as the internal control.

Immunofluorescence staining. Following exposure to the aforementioned treatments, HCMs were washed with PBS three times and fixed with $100 \%$ methanol for $10 \mathrm{~min}$ at room temperature. Next, cells were washed three times with PBS and permeabilized with $1 \%$ Triton X-100 (Sigma-Aldrich; Merck KGaA) for $10 \mathrm{~min}$ at room temperature. The cells were subsequently blocked with 4\% BSA (Sigma-Aldrich; Merck KGaA) in PBS for $1 \mathrm{~h}$ at room temperature and incubated with primary antibodies against Ki67 $(1 \mu \mathrm{g} / \mathrm{ml}$; cat. no. ab15580; Abcam) or LC3 (1 $\mu \mathrm{g} / \mathrm{ml}$; cat. no. ab192890;
Abcam) for $2 \mathrm{~h}$ at $4^{\circ} \mathrm{C}$. Following primary antibody incubation, cells were incubated with a FITC-conjugated anti-rabbit IgG secondary antibody (1:5,000; cat. no. 150077; Abcam) for $1 \mathrm{~h}$ at room temperature. Finally, the cells were washed three times with PBS and stained with DAPI (Vector Laboratories, Inc.) for $5 \mathrm{~min}$ at room temperature. The cells were imaged using a fluorescence microscope (magnification, x200) and the number of nuclei and Ki67-postive cells were counted in three randomly-selected fields.

Monodansylcadaverine (MDC) staining. A total of $2 \times 10^{5} \mathrm{HCMs}$ were seeded in a 6 -well plate and then treated with different concentrations of TZM or a combination of TZM and ginsenoside $\mathrm{Rg} 2$. Cells were then labeled with MDC $(50 \mu \mathrm{M})$ in PBS for $10 \mathrm{~min}$ at $37^{\circ} \mathrm{C}$ in the dark. After washing with PBS three times, cells were fixed in 4\% PFA for $30 \mathrm{~min}$ at room temperature. Subsequently, the cells were visualized under a fluorescence microscope (magnification, x200).

Statistical analysis. Statistical analyses were performed using SPSS software (version 17.0; SPSS, Inc.). Data are expressed as the mean \pm SD of three replicates. Comparisons among multiple groups were made with the one-way ANOVA followed by Tukey's post hoc test. $\mathrm{P}<0.05$ was considered to indicate a statistically significant difference.

\section{Results}

TZM inhibits the proliferation of HCMs by inhibiting the Akt/mTOR signaling pathway. HCMs were treated with increasing concentrations of TZM $(0,10,50,100$ or $200 \mu \mathrm{g} / \mathrm{ml}$ ) for $24 \mathrm{~h}$, and the effects of TZM on cell proliferation were measured using a CCK- 8 assay. The results revealed that 50,100 or $200 \mu \mathrm{g} / \mathrm{ml}$ TZM significantly decreased cell proliferation compared with the control group (Fig. 1A). These data suggested that TZM inhibited the proliferation of HCMs in a dose-dependent manner, with an $\mathrm{IC}_{50}$ value of $88 \mu \mathrm{g} / \mathrm{ml}$. Annexin V/PI staining was subsequently performed to determine the percentage of apoptotic cells following treatment with 50 or $100 \mu \mathrm{g} / \mathrm{ml}$ TZM. As indicated in Fig. 1B and C, a significant increase in apoptotic cells was observed in TZM-treated cells compared with the controls. Furthermore, western blotting revealed that the levels of p-Akt and p-mTOR in cells were significantly decreased following treatment with TZM compared with controls (Fig. 1D-F). Therefore, $100 \mu \mathrm{g} / \mathrm{ml}$ TZM was used for subsequent experiments. These results suggested that TZM could induce apoptosis of HCMs by inhibiting the Akt/mTOR signaling pathway.

TZM-induced cytotoxicity in HCMs is reversed by ginsenoside $\mathrm{Rg} 2$. The chemical structure of ginsenoside $\mathrm{Rg} 2$ is presented in Fig. 2A. A CCK-8 assay was performed to evaluate the effects of ginsenoside $\mathrm{Rg} 2$ or the combination of ginsenoside Rg2 and TZM on HCM proliferation. The results indicated that ginsenoside $\mathrm{Rg} 2$ (at concentrations between $0-200 \mu \mathrm{M}$ ) exhibited no cytotoxicity (Fig. 2B). Therefore, $200 \mu \mathrm{M}$ ginsenoside $\mathrm{Rg} 2$ was used for subsequent experiments. Pretreatment with ginsenoside $\mathrm{Rg} 2$ significantly reversed TZM-induced cytotoxicity compared with TZM alone (Fig. 2C). 
A

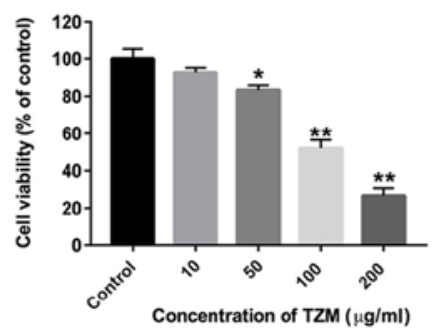

D

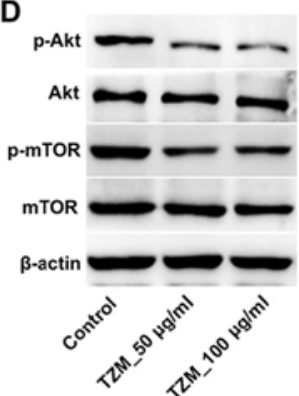

B

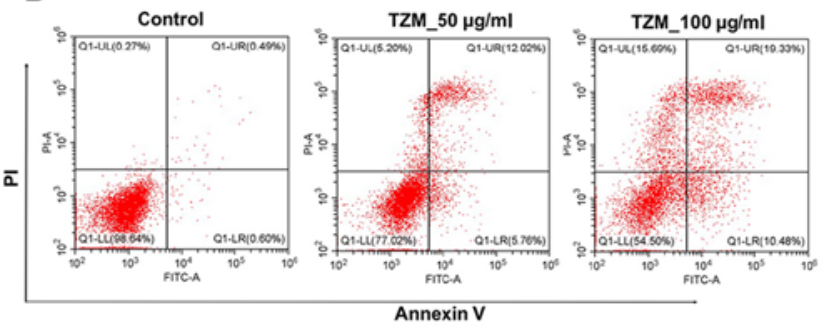

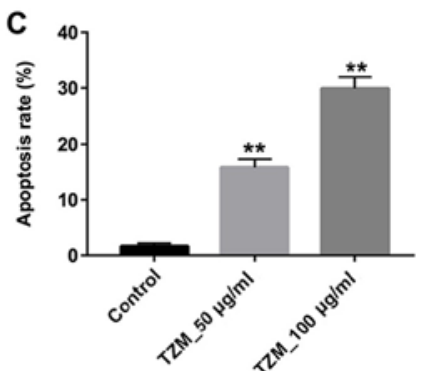

E

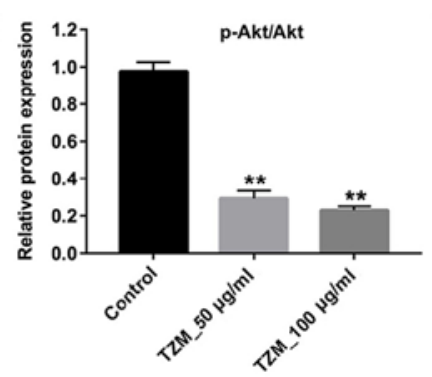

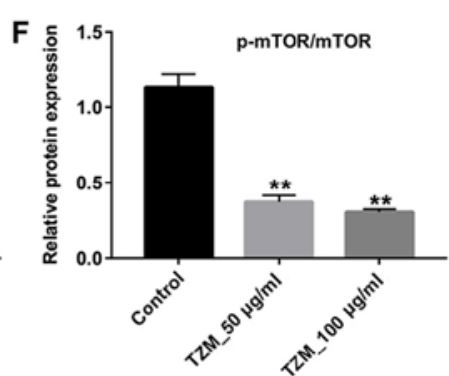

Figure 1. TZM inhibits the proliferation of HCMs by inhibiting the Akt/mTOR signaling pathway. (A) HCMs were treated with different concentrations of TZM $(0,10,50,100$ or $200 \mu \mathrm{g} / \mathrm{ml})$ for $24 \mathrm{~h}$, and cell proliferation was examined using a Cell Counting Kit- 8 assay. (B) HCMs were incubated with 0,50 or $100 \mu \mathrm{g} / \mathrm{ml} \mathrm{TZM}$ for $24 \mathrm{~h}$, and apoptotic cells were detected with Annexin V/PI staining and flow cytometry. (C) Quantification of Annexin V-positive cells. (D) Cells were treated with 0,50 or $100 \mu \mathrm{g} / \mathrm{ml} \mathrm{TZM}$ for $24 \mathrm{~h}$, and the expression of p-Akt and p-mTOR protein was measured by western blotting. The relative levels of (E) p-Akt and (F) p-mTOR were normalized to Akt and mTOR, respectively and quantified using ImageJ software. Data were representative of three separate experiments. ${ }^{*} \mathrm{P}<0.05$ and ${ }^{* *} \mathrm{P}<0.01$ vs. the control group. TZM, trastuzumab; HCM, human cardiomyocyte; PI, propidium iodide; p, phosphorylated.

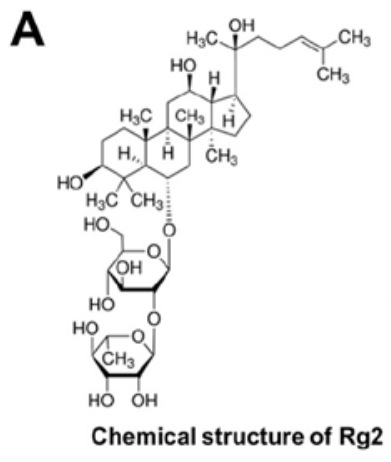

D

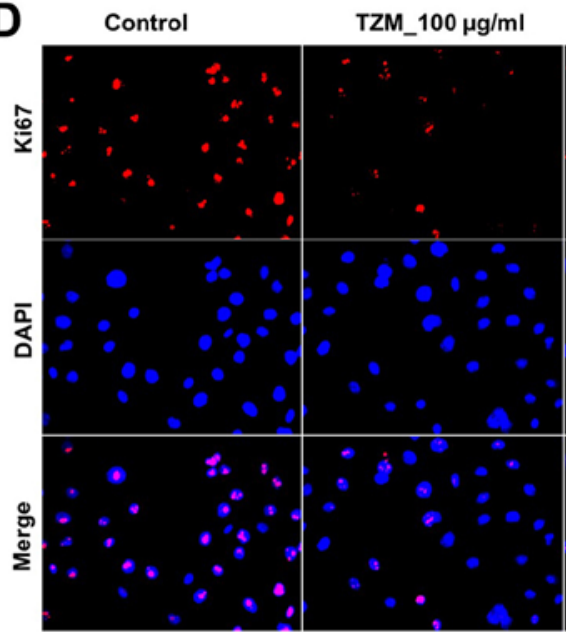

B

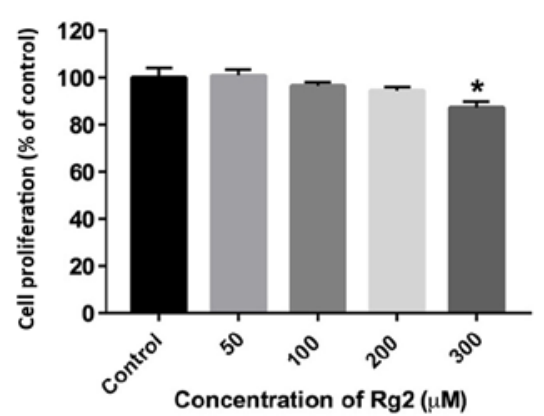

Rg2_200 $\mu \mathrm{M}$

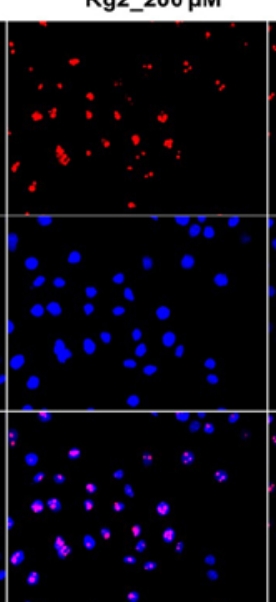

$\mathrm{Rg} 2+\mathrm{TZM}$

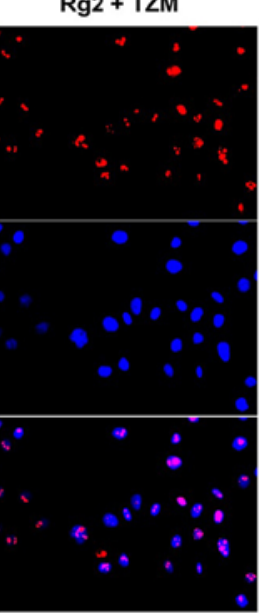

C
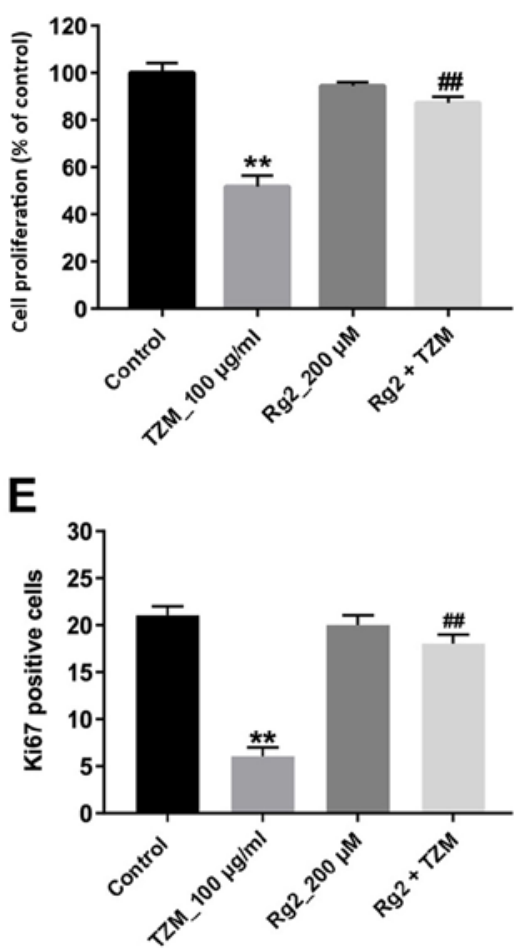

Figure 2. TZM-induced cytotoxicity is reversed by ginsenoside Rg2. (A) Chemical structure of ginsenoside Rg2. (B) HCMs were incubated with $0,50,100,200$ or $300 \mu \mathrm{M}$ ginsenoside $\mathrm{Rg} 2$ for $12 \mathrm{~h}$, and cell proliferation was examined with a CCK-8 assay. (C) HCMs were incubated with ginsenoside Rg2 and/or TZM at the indicated time points and cell proliferation was assessed using a CCK-8 assay. (D) Cell proliferation was detected by immunofluorescence staining with DAPI and Ki67. Representative micrographs of Ki67-positive HCMs (red) and DAPI staining (blue) of the nuclei. magnification, x200 (E) The percentage of Ki67-positive HCMs was calculated from three randomly-captured images for each group after treatment. " $\mathrm{P}<0.05$ and ${ }^{* *} \mathrm{P}<0.01$ vs. the control group; ${ }^{\# \#} \mathrm{P}<0.01$ vs. the TZM group. TZM, trastuzumab; HCM, human cardiomyocyte; CCK-8, Cell Counting Kit-8; Rg2, ginsenoside Rg2. 
A

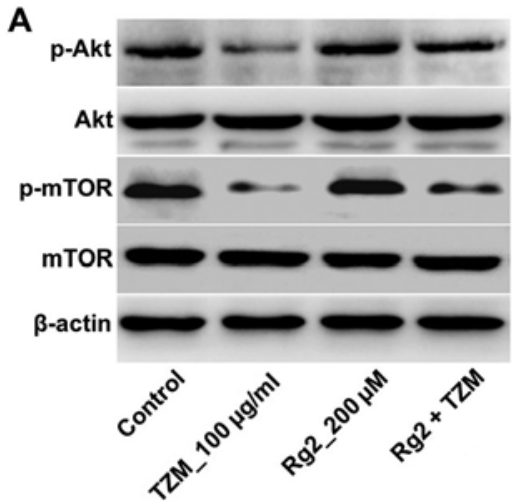

B

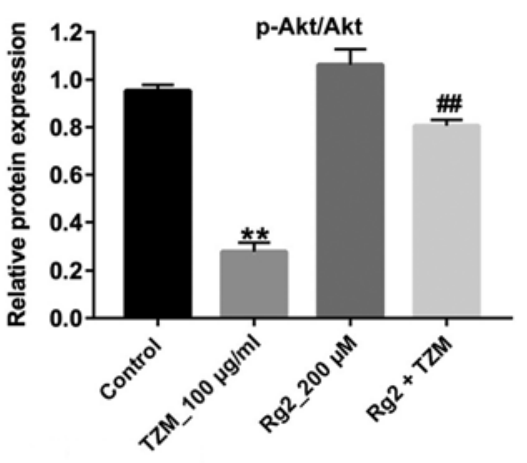

C

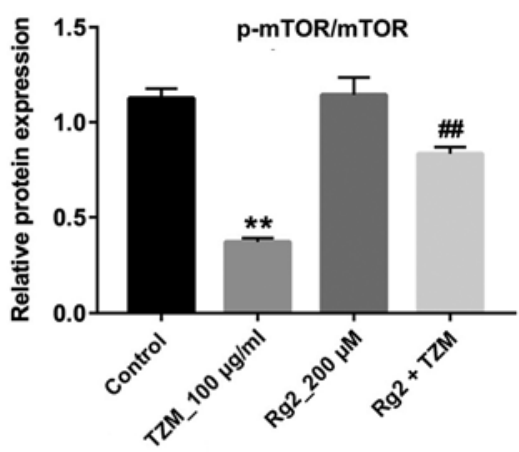

Figure 3. Akt/mTOR pathway inhibition effect of TZM is reversed by ginsenoside $\mathrm{Rg} 2$. (A) HCMs were incubated with $200 \mu \mathrm{M}$ ginsenoside Rg2 for $12 \mathrm{~h}$ and exposed to $100 \mu \mathrm{g} / \mathrm{ml} \mathrm{TZM}$ for another $24 \mathrm{~h}$. The expression of p-Akt and p-mTOR in cells was measured by western blotting. The relative levels of (B) p-Akt and (C) p-mTOR were normalized to Akt and mTOR, respectively and quantified using ImageJ software. Data are representative of three independent experiments. ${ }^{* *} \mathrm{P}<0.01$ vs. the control group; ${ }^{\# \#} \mathrm{P}<0.01$ vs. the TZM group. TZM, trastuzumab; HCM, human cardiomyocyte; p, phosphorylated; Rg2, ginsenoside Rg2.

A

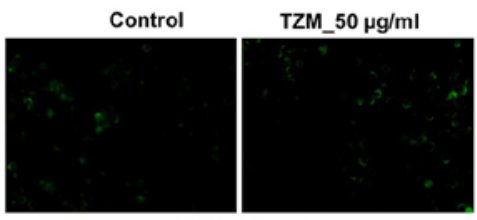

TZM_100 $\mu \mathrm{g} / \mathrm{ml}$

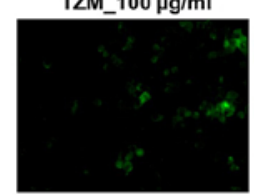

D

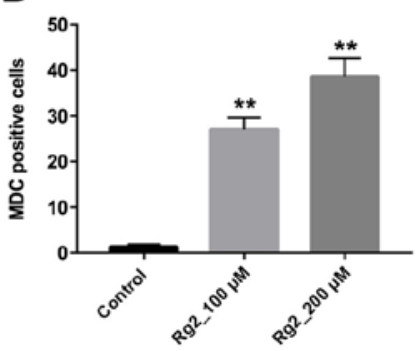

E

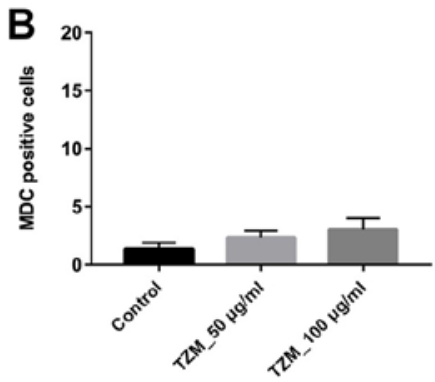

C
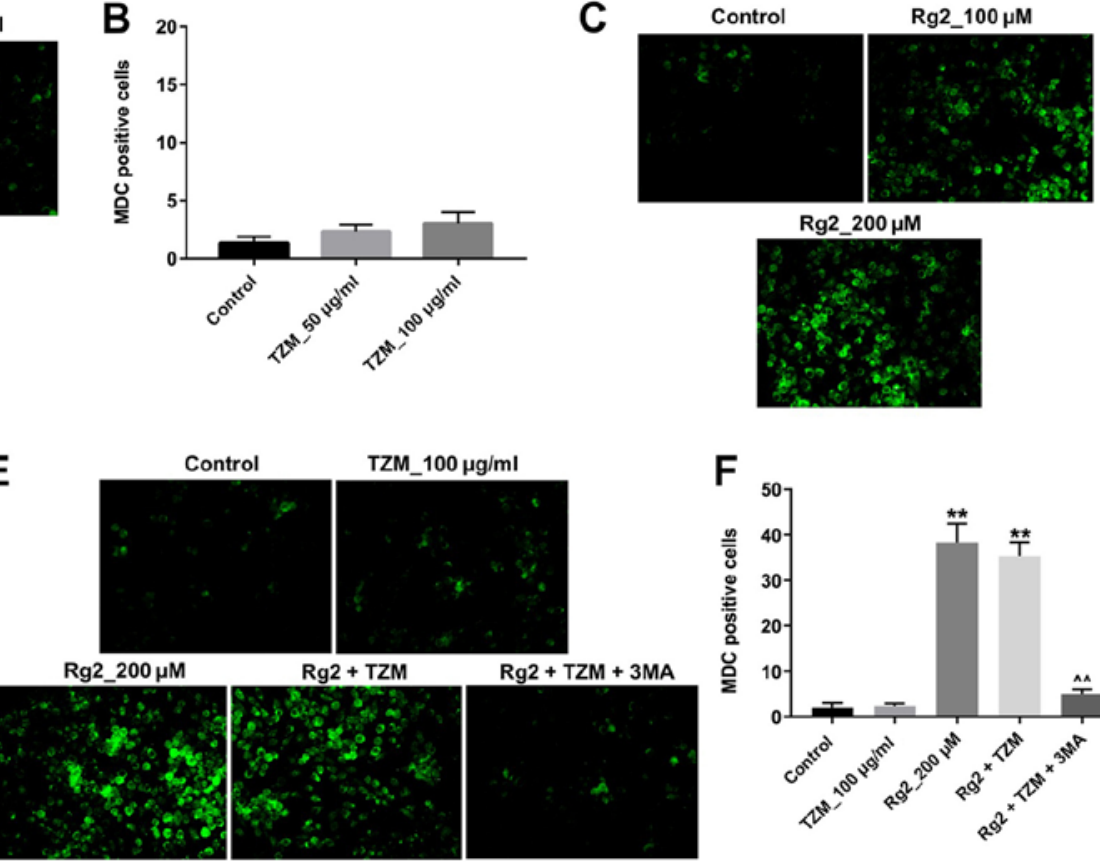

Control

TZM_100 $\mu \mathrm{g} / \mathrm{ml}$

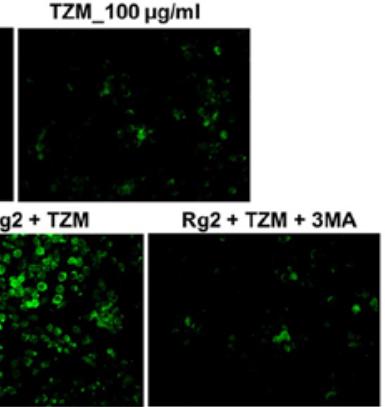

F

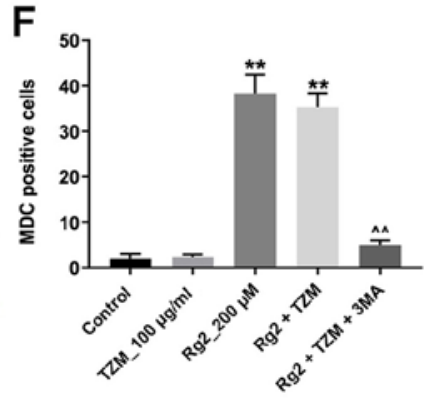

Figure 4. Ginsenoside Rg2 induces autophagy of HCMs. (A) HCMs were labeled with MDC after incubation with 0,50 or $100 \mu \mathrm{g} / \mathrm{ml}$ TZM for $24 \mathrm{~h}$. Magnification, $\mathrm{x} 200$. (B) Quantitative analysis of MDC-positive cells in (A). (C) HCMs were labeled with MDC after incubation with 0 , 100 or $200 \mu \mathrm{M}$ ginsenoside Rg2 for $12 \mathrm{~h}$. Magnification, x200. (D) Quantitative analysis of MDC-positive cells in (C). (E) HCMs were exposed to $200 \mu \mathrm{M}$ ginsenoside Rg2 with or without $5 \mathrm{mM} 3-\mathrm{MA}$ for $12 \mathrm{~h}$ and exposed to $100 \mu \mathrm{g} / \mathrm{ml}$ TZM for another $24 \mathrm{~h}$. Cell autophagy was then detected with MDC staining. Magnification, x200. (F) Quantitative analysis of MDC-positive cells from. (E) Data are representative of three independent experiments. ${ }^{* *} \mathrm{P}<0.01$ vs. the control group. ${ }^{\wedge} \wedge \mathrm{P}<0.01$ vs. the Rg2 + TZM group. TZM, trastuzumab; HCM, human cardiomyocyte; MDC, monodansylcadaverine; 3-MA, 3-methyladenine; Rg2, ginsenoside Rg2.

HCMs were subsequently subjected to immunofluorescence staining. Ki67 expression was significantly increased in the ginsenoside Rg2-pretreated group compared with the TZM group (Fig. 2D and E). Furthermore, TZM-induced p-Akt and p-mTOR downregulation was significantly reversed by ginsenoside Rg2 compared with the TZM group (Fig. 3A-C). Collectively, these results suggested that ginsenoside $\mathrm{Rg} 2$ may significantly alleviate TZM-induced cardiotoxicity by upregulating the Akt/mTOR signaling pathway.

Ginsenoside Rg2 induces autophagy of HCMs. MDC is a selective fluorescent marker that labels autophagic vacuoles (11).
To further explore the mechanisms underlying the protective effect of ginsenoside Rg2 against TZM cytotoxicity, MDC fluorescence staining was performed. The results revealed that MDC fluorescence was barely detected in HCMs incubated with TZM (Fig. 4A and B), whereas strong MDC fluorescence was observed in ginsenoside Rg2-treated cells (Fig. 4C and D). Furthermore, the combination of ginsenoside Rg2 and TZM significantly increased the levels of MDC fluorescence compared with the control group, which were subsequently significantly decreased in the presence of the autophagy inhibitor 3-MA (Fig. 4E and F). Therefore, the results suggested that ginsenoside Rg2 may significantly induce autophagy in HCMs. 

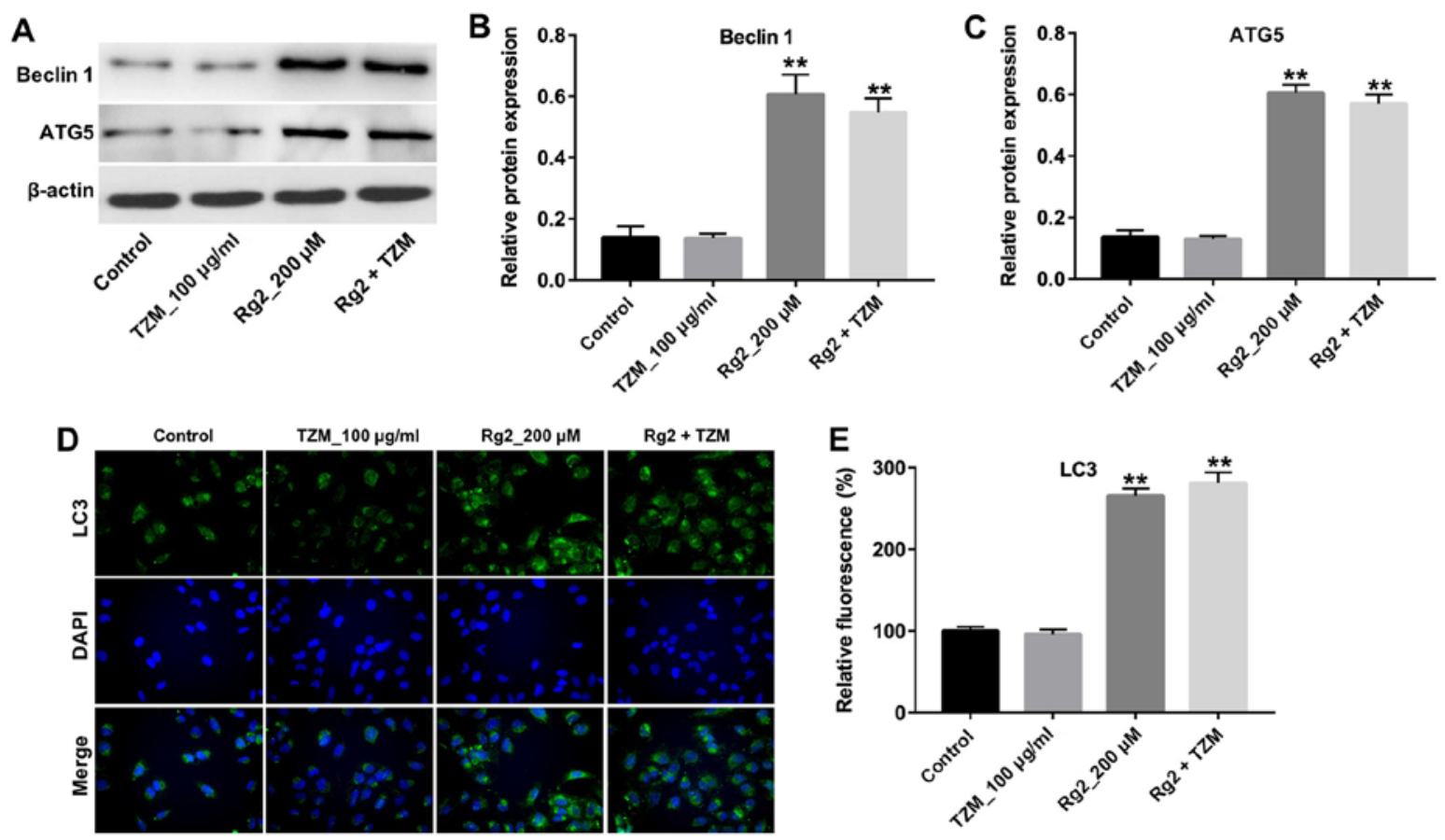

Figure 5. Ginsenoside $\operatorname{Rg} 2$ increases the expression of autophagy-related proteins beclin 1, LC3 and ATG5. (A) HCM cells were incubated with $200 \mu \mathrm{M}$ ginsenoside Rg2 for $12 \mathrm{~h}$ and exposed to $100 \mu \mathrm{g} / \mathrm{ml}$ TZM for another $24 \mathrm{~h}$. The expression levels of beclin 1, LC3 and ATG5 were measured by western blotting. The relative levels of (B) beclin 1 and (C) ATG5 were normalized to $\beta$-actin and quantified using ImageJ software. (D) The expression of LC3 in cells was detected with immunofluorescence staining. Magnification, x200. (E) Quantification of LC3-positive cells. Data were representative of three separate experiments. ${ }^{* *} \mathrm{P}<0.01$ vs. the control group. TZM, trastuzumab; HCM, human cardiomyocyte; LC3, microtubule associated protein 1 light chain $3 \alpha$; ATG5, autophagy protein 5; $\mathrm{Rg} 2$, ginsenoside $\mathrm{Rg} 2$.
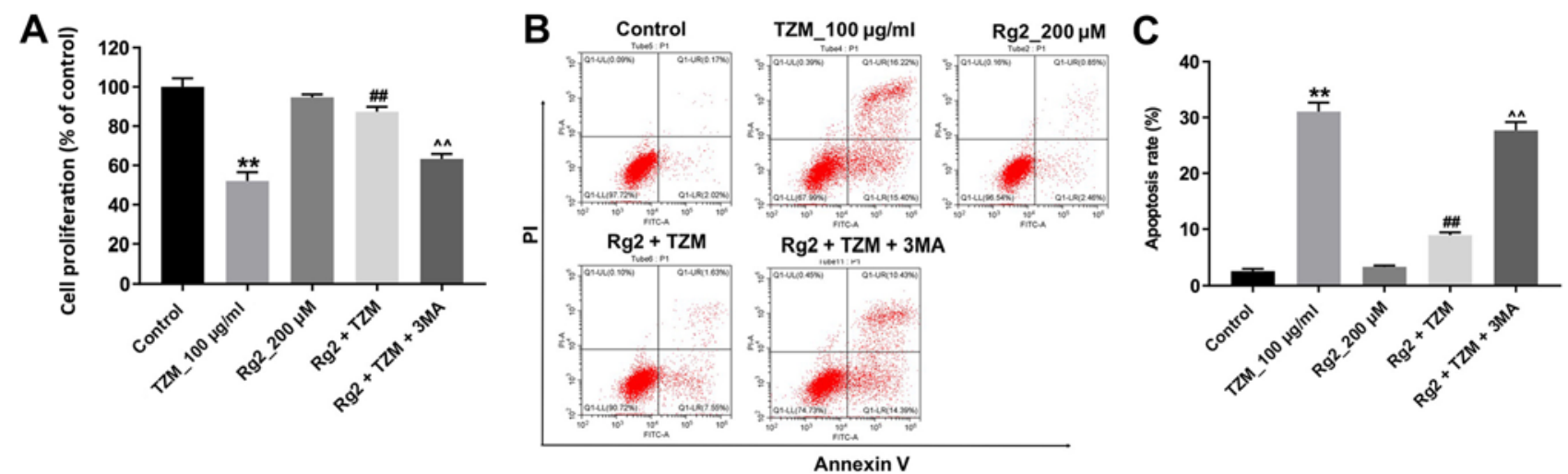

Figure 6. Inhibition of autophagy abolishes the protective effect of ginsenoside Rg2 against TZM in HCMs. (A) HCMs were exposed to $200 \mu \mathrm{M}$ ginsenoside Rg2 with or without $5 \mathrm{mM}$ 3-MA for $12 \mathrm{~h}$ and exposed to $100 \mu \mathrm{g} / \mathrm{ml}$ TZM for another $24 \mathrm{~h}$. Cell proliferation was examined with a Cell Counting Kit-8 assay. (B) Apoptotic cells were detected using Annexin V/PI staining. (C) Quantification of apoptotic cells. ${ }^{* *} \mathrm{P}<0.01$ vs. the control group; ${ }^{\# \#} \mathrm{P}<0.01$ vs. the TZM group; $\wedge \wedge \mathrm{P}<0.01$ vs. the Rg2 + TZM group. TZM, trastuzumab; HCM, human cardiomyocyte; 3-MA, 3-methyladenine; PI, propidium iodide; Rg2, ginsenoside $\mathrm{Rg} 2$.

Ginsenoside Rg2 increases the expression of the autophagy-related proteins beclin 1, LC3 and ATG5 in $H C M s$. To further explore the mechanisms underlying ginsenoside Rg2-induced autophagy, the expression of the key autophagy markers beclin 1, LC3 and ATG5 was detected by western blotting. As indicated in Fig. 5A-C, treatment with TZM did not significantly affect the expression of beclin 1 and ATG5 in cells compared with controls; however, the levels of beclin1 and ATG5 were significantly increased in ginsenoside $\mathrm{Rg} 2$-treated cells compared with controls. In addition, the results of immunofluorescence staining indicated the nuclear expression of LC3 was significantly increased following $\mathrm{Rg} 2$ treatment (Fig. 5D and E). These data further demonstrated that ginsenoside $\operatorname{Rg} 2$ increased autophagy in HCMs by increasing the expression of the autophagy-related proteins beclin 1, LC3 and ATG5.

Inhibition of autophagy in HCMs abolished the protective effect of ginsenoside Rg2 against TZM. To verify whether ginsenoside $\mathrm{Rg} 2$ exerted a protective effect against TZM in HCMs by inducing autophagy, the effects of the autophagy inhibitor 3-MA were investigated. As indicated in Fig. 6A, the 
protective effects of ginsenoside $\mathrm{Rg} 2$ against TZM-induced cytotoxicity in HCMs were reversed by 3-MA. Meanwhile, the antiapoptotic effects of ginsenoside $\mathrm{Rg} 2$ in TZM-stimulated HCMs were also alleviated by 3-MA (Fig. 6B and C). These data indicated that ginsenoside $\operatorname{Rg} 2$ could attenuate TZM-induced cytotoxicity in HCMs by inducing autophagy.

\section{Discussion}

The present study established an in vitro model of TZMinduced toxicity in HCMs. TZM decreased cell proliferation, increased apoptosis and decreased the expression of p-Akt and p-mTOR. However, TZM-induced cytotoxicity and p-Akt and p-mTOR downregulation were reversed by ginsenoside $\mathrm{Rg} 2$. In addition, ginsenoside $\mathrm{Rg} 2$ significantly induced autophagy in HCMs by increasing the levels of beclin 1, LC3 and ATG5. The present study revealed that ginsenoside $\mathrm{Rg} 2$ may protect HCMs from TZM-induced toxicity by activating autophagy.

It was hypothesized that ginsenoside $\operatorname{Rg} 2$ has antioxidant, antidiabetic, antiapoptotic and neuroprotective activities $(3,12,13)$. A previous study reported that ginsenoside $\mathrm{Rg} 2$ exerted protective effects against hydrogen peroxide-induced injury and apoptosis in HCMs (6). Furthermore, ginsenoside $\operatorname{Rg} 2$ increased autophagy and activated the p53/AMPK signaling pathway in MCF-7 breast cancer cells (5). Moreover, ginsenoside $\mathrm{Rg} 2$ decreased lipopolysaccharide-induced Bax and caspase- 3 and -9 expression, and exerted an antiapoptotic effect in neurons (14). Kang et al (15) found that ginsenoside $\mathrm{Rg} 2$ could protect $\mathrm{HaCaT}$ cells from UV-B-induced cell damage. Meanwhile, Wang et al (16) indicated that ginsenoside $\mathrm{Rg} 2$ could inhibit the proliferation of breast cancer cells in vitro. The aforementioned studies indicated that ginsenoside $\operatorname{Rg} 2$ exhibited dual functions in protecting cells from DNA damage and inducing apoptosis in cancer cells. The present study investigated whether pretreatment with ginsenoside $\mathrm{Rg} 2$ could reverse TZM-induced toxicity in HCMs. The results revealed that ginsenoside $\mathrm{Rg} 2$ exhibited protective effects against TZM-induced toxicity by inducing autophagy in HCMs. Moreover, to the best of our knowledge, the present study was the first to demonstrate that ginsenoside $\mathrm{Rg} 2$ exhibited protective effects against TZM-induced toxicity in HCMs.

Beclin 1, LC3 and ATG5 are autophagy-related proteins required for autophagosome elongation (17). In the present study, pretreatment with ginsenoside $\mathrm{Rg} 2$ increased the levels of beclin 1, LC3 and ATG5, which indicated that TZM-induced toxicity was reversed by ginsenoside $\mathrm{Rg} 2$ by increasing autophagy. A previous study revealed that $\mathrm{p}-53$ and $\mathrm{p}$-AMPK were involved in ginsenoside $\mathrm{Rg} 2$-induced autophagy in MCF-7 cells (5). However, in the present study, pretreatment with ginsenoside $\mathrm{Rg} 2$ reversed TZM-induced downregulation of p-Akt and p-mTOR in HCMs. It was reported that canonical autophagy requires mTOR inhibition (18). Moreover, the Akt/mTOR signaling pathway is one of the main downstream effectors of HER2 (19). Previous studies demonstrated that TZM exerted antitumor activities by inhibiting the Akt/mTOR signaling pathway in HER2-overexpressing breast cancer cells $(20,21)$. In the present study, treatment with ginsenoside $\mathrm{Rg} 2$ alone had no effect on the Akt/mTOR signaling pathway in HCMs. The inhibition of the Akt/mTOR signaling pathway induced by TZM was reversed by ginsenoside Rg2. Evidence has shown that the AKT/mTOR pathway plays an important role in several cellular processes, including proliferation, survival and autophagy (22). Hu et al (23) found that inhibition of autophagy promoted advanced glycation end product-induced apoptosis in cardiomyocytes by inhibiting the AKT/mTOR signaling. In the present study, TZM-induced p-Akt and p-mTOR protein decreases were significantly reversed by ginsenoside $\mathrm{Rg} 2$ treatment, indicating that ginsenoside $\mathrm{Rg} 2$ could induce autophagy of HCM cells by activating the AKT/mTOR pathway. Meanwhile, the anti-apoptotic effects of ginsenoside Rg2 in TZM-stimulated HCMs cells were reversed by 3-MA treatment, indicating that inhibition of autophagy abolished the protective effect of ginsenoside $\mathrm{Rg} 2$ against TZM in HCMs. The results suggested that the mechanism by which ginsenoside $\operatorname{Rg} 2$ protected against TZM-induced cardiotoxicity was reversed by inhibition of the Akt/mTOR signaling pathway.

Collectively, the results obtained in the present study revealed that ginsenoside $\mathrm{Rg} 2$ exhibited protective effects against TZM-induced toxicity in HCMs by activating autophagy and increasing the expression of Akt and mTOR. Ginsenoside Rg2 may serve as a potential clinical agent to prevent TZM-related cardiotoxicity and may provide significant beneficial effects for patients with breast cancer. However, the data presented in this study requires further in vivo validation prior to the clinical application of ginsenoside $\mathrm{Rg} 2$.

\section{Acknowledgements}

Not applicable.

\section{Funding}

No funding was received.

\section{Availability of data and materials}

The datasets used and/or analyzed during the current study are available from the corresponding author on reasonable request.

\section{Authors' contributions}

GL made major contributions to the conception, design and manuscript drafting of this study. GL, XL and FS were responsible for data acquisition, data analysis, data interpretation and manuscript revision. XQ made substantial contributions to conception and design of the study and revised the manuscript. All authors agreed to be accountable for all aspects of the work. All authors read and approved the final manuscript.

\section{Ethics approval and consent to participate}

Not applicable.

\section{Patient consent for publication}

Not applicable. 


\section{Competing interests}

The authors declare that they have no competing interests.

\section{References}

1. Xu QM, Jia D, Gao HW, Zhang MM, He WJ, Pan S, Liu YL, Li XR, Cui JH and Yang SL: In vitro and in vivo protective effects of gingenosides on acute renal injury induced by cantharidin. J Functional Foods 5: 2012-2018, 2013.

2. Chen XJ, Zhang XJ, Shui YM, Wan JB and Gao JL: Anticancer activities of protopanaxadiol- and protopanaxatriol-type ginsenosides and their metabolites. Evid Based Complement Alternat Med 2016: 5738694, 2016.

3. Zhang G, Liu A, Zhou Y, San X, Jin T and Jin Y: Panax ginseng ginsenoside- $\mathrm{Rg} 2$ protects memory impairment via anti-apoptosis in a rat model with vascular dementia. J Ethnopharmacol 115: 441-448, 2008

4. Fan Y, Wang N, Rocchi A, Zhang W, Vassar R, Zhou Y and He C: Identification of natural products with neuronal and metabolic benefits through autophagy induction. Autophagy 13: 41-56, 2017.

5. Chung Y, Jeong S, Choi HS, Ro S, Lee JS and Park JK: Upregulation of autophagy by Ginsenoside Rg2 in MCF-7 cells Anim Cells Syst (Seoul) 22: 382-389, 2018.

6. Fu W, Sui D, Yu X, Gou D, Zhou Y and Xu H: Protective effects of ginsenoside $\mathrm{Rg} 2$ against $\mathrm{H}_{2} \mathrm{O}_{2}$-induced injury and apoptosis in H9c2 cells. Int J Clin Exp Med 8: 19938-19947, 2015.

7. Gershon N, Berchenko Y, Hall PS and Goldstein DA: Cost effectiveness and affordability of trastuzumab in sub-Saharan Africa for early stage. Cost Eff Resour Alloc 17: 5, 2019.

8. Slamon D, Eiermann W, Robert N, Pienkowski T, Martin M, Press M, Mackey J, Glaspy J, Chan A, Pawlicki M, et al: Adjuvant trastuzumab in HER2-positive breast cancer. N Engl J Med 365: $1273-1283,2011$.

9. Mazzotta M, Krasniqi E, Barchiesi G, Pizzuti L, Tomao F, Barba $\mathrm{M}$ and Vici P: Long-term safety and real-world effectiveness of trastuzumab in breast cancer. J Clin Med 8: 254, 2019.

10. Sato A, Yoshihisa A, Miyata-Tatsumi M, Oikawa M, Kobayashi A, Ishida T, Ohtake T and Takeishi Y: Valvular heart disease as a possible predictor of trastuzumab-induced cardiotoxicity in patients with breast cancer. Mol Clin Oncol 10: 37-42, 2019.

11. Biederbick A, Kern HF and Elsässer HP: Monodansylcadaverine (MDC) is a specific in vivo marker for autophagic vacuoles. Eur J Cell Biol 66: 3-14, 1995.

12. Jeong SJ, Han SH, Kim DY, Lee JC, Kim HS, Kim BH, Lee JS, Hwang EH and Park JK: Effects of $\mathrm{mRg} 2$, a mixture of ginsenosides containing $60 \% \mathrm{Rg} 2$, on the ultraviolet B-induced DNA repair synthesis and apoptosis in NIH3T3 cells. Int J Toxicol 26 151-158, 2007.
13. Ye J, Yao JP, Wang X, Zheng M, Li P, He C, Wan JB, Yao X and $\mathrm{Su} \mathrm{H}$ : Neuroprotective effects of ginsenosides on neural progenitor cells against oxidative injury. Mol Med Rep 13: 3083-3091, 2016

14. Chung YH, Jeong SA, Choi HS, Ro S, Lee JS and Park JK: Protective effects of ginsenoside Rg2 and astaxanthin mixture against UVB-induced DNA damage. Anim Cells Syst (Seoul) 22: 400-406, 2018.

15. Kang HJ, Huang YH, Lim HW, Shin D, Jang K, Lee Y, Kim K and Lim CJ: Stereospecificity of ginsenoside Rg2 epimers in the protective response against UV-B radiation-induced oxidative stress in human epidermal keratinocytes. J Photochem Photobiol B 165: 232-239, 2016.

16. Wang CZ, Aung HH, Zhang B, Sun S, Li XL, He H, Xie JT, $\mathrm{He}$ TC, Du W and Yuan CS: Chemopreventive effects of heat-processed Panax quinquefolius root on human breast cancer cells. Anticancer Res 28: 2545-2551, 2008.

17. Mondaca-Ruff D, Riquelme JA, Quiroga C, Norambuena-Soto I, Sanhueza-Olivares F, Villar-Fincheira P, Hernández-Díaz T, Cancino-Arenas N, San Martin A, García L, et al: Angiotensin II-regulated autophagy is required for vascular smooth muscle cell hypertrophy. Front Pharmacol 9: 1553, 2019.

18. Gatica D, Chiong M, Lavandero S and Klionsky DJ: Molecular mechanisms of autophagy in the cardiovascular system. Circ Res 116: 456-467, 2015.

19. Sukawa Y, Yamamoto H, Nosho K, Ito M, Igarashi H, Naito T, Mitsuhashi K, Matsunaga Y, Takahashi T, Mikami M, et al: HER2 expression and PI3K-Akt pathway alterations in gastric cancer. Digestion 89: 12-17, 2014.

20. Yang Y, Ren F, Tian Z, Song W, Cheng B and Feng Z: Osthole synergizes with HER2 inhibitor, trastuzumab in HER2-overexpressed N87 Gastric cancer by inducing apoptosis and inhibition of AKT-MAPK pathway. Front Pharmacol 9: 1392,2018

21. Han S, Meng Y, Tong Q, Li G, Zhang X, Chen Y, Hu S, Zheng L, Tan W, Li H, et al: The ErbB2-targeting antibody trastuzumab and the small-molecule SRC inhibitor saracatinib synergistically inhibit ErbB2-overexpressing gastric cancer. MAbs 6: 403-408, 2014.

22. Yu JS and Cui W: Proliferation, survival and metabolism: The role of PI3K/AKT/mTOR signalling in pluripotency and cell fate determination. Development 143: 3050-3060, 2016.

23. Hu P, Zhou H, Lu M, Dou L, Bo G, Wu J and Huang S: Autophagy plays a protective role in advanced glycation end product-induced apoptosis in cardiomyocytes. Cell Physiol Biochem 37: 697-706, 2015.

This work is licensed under a Creative Commons Attribution-NonCommercial-NoDerivatives 4.0 International (CC BY-NC-ND 4.0) License. 